\title{
Detecting Objects Thrown over Fence in Outdoor Scenes
}

\author{
Róbert Csordás ${ }^{1,2}$, László Havasi ${ }^{1}$ and Tamás Szirányi ${ }^{1,2}$ \\ ${ }^{1}$ MTA SZTAKI, Kende u. 13-17, Budapest, Hungary \\ ${ }^{2}$ Budapest University of Technology and Economics, Müegyetem rkp. 3, Budapest, Hungary
}

Keywords: Optical Flow, Trajectory Matching, Computer Vision, Surveillance System.

\begin{abstract}
We present a new technique for detecting objects thrown over a critical area of interest in a video sequence made by a monocular camera. Our method was developed to run in real time in an outdoor surveillance system. Unlike others, we use an optical flow based motion detection and tracking system to detect the object's trajectories and for parabolic path search. The system successfully detects thrown objects of various sizes and is unaffected by the rotation of the objects.
\end{abstract}

\section{INTRODUCTION}

Moving object detection is widely used in surveillance systems. We introduce a robust method which uses a monocular camera system for detecting objects thrown over a fence in outdoor environments.

The challenge in developing such a method for outdoor scenes is to detect object trajectories reliably and to filter out motions that are irrelevant, such as a person walking or a waving branch of a tree. The thrown object can be small and blurry, causing some of the feature extraction based tracking algorithms to perform poorly.

The method described here is designed to work with a specific camera placement. The camera should be placed at the end of the fence, and it should be pointed exactly to the line of the fence. This would make the camera capture a whole and clean image of the area of interest. This criterion is easily met in real world surveillance scenarios.

Detecting thrown objects by looking at their trajectories can be accomplished easily. They always follow a parabolic path, even after perspective transformation. After the filtering of the parameters (like size, length and slope) of the detected parabolic trajectories, the thrown objects can be identified.

Unlike other research in the field, we use optical flow for finding object trajectories instead of tracking points of interest. This method is more reliable for tracking objects that are blurry, small, or have a variable shape. It is also immune to object rotation around the axis in the image plane that makes the points of interest disappear and re-appear. It can be easily used to track multiple objects, and it is robust against motions that are not interfering with the position of the tracked object.

\subsection{Related Work}

There are several systems with the capability of detecting thrown objects. These systems are commercial, and their theory of operation is hardly known. There are very little publicly available studies in this field; most of the methods are based on motion detection over a dedicated zone, and the path of motion is not considered. The most related study is (Ribnick et al., 2007). The authors use an inter-frame differentiating method for identifying areas of rapid motion in the image. They filter them in advanced ways, keeping track of their centroids, and use expectation maximization to find parabolic trajectories. Our method uses a different way of object tracking which is more suitable for outdoor use and a different method for trajectory matching.

The method described in (Venetianer et al., 2005) uses interesting point detection based object tracking methods and measures of salience that are not related to the parabolic trajectories of falling objects.

We use ideas described in (Denman et al., 2009) to improve the performance and robustness of the algorithm. We use background segmentation methods to determine the areas of interest, and we run optical flow on these areas. These areas are usually much smaller than the area of possible actions, thus reducing the resources needed for optical flow computation. The background segmentation also filters out some 


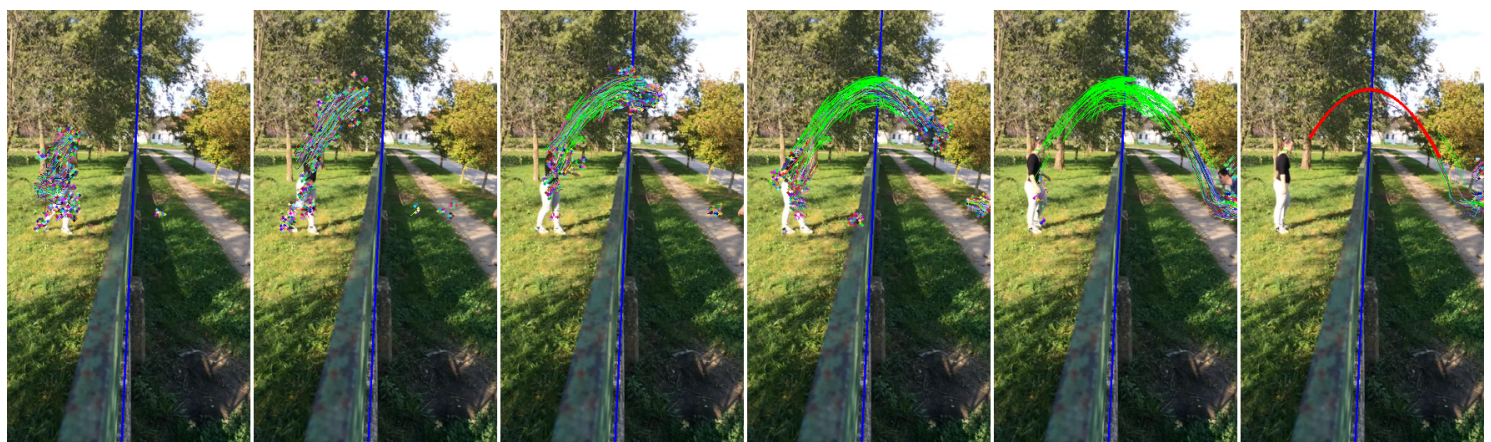

Figure 1: The sequence of a throw and the output of the algorithm.

types of background clutter, which can otherwise interfere with the optical flow algorithm.

\section{PROPOSED METHOD}

The goal of the project was to reliably detect objects thrown over a fence. The object can be deformable, and the algorithm must work in real world applications. Our method consists of four major steps: frame capturing and preprocessing, moving point detection, tracking points, and trajectory matching. The structure of the system is shown on Figure 4. An example sequence with the output of the algorithm is shown on Figure 1.

\subsection{Frame Capturing and Preprocessing}

The frame captured by camera is needed to be preprocessed. Firstly, it is transformed to monochrome image. The image size is scaled down to size enough for the accurate operation of the algorithm but yet it is less resource hungry. The scale is selected so that the algorithm performs reasonably fast (360x640 pixels). A Gaussian blur is applied to the resulting image. This makes the algorithm more immune to noise and makes optical flow algorithm perform better.

\subsection{Moving Point Detection}

After the preprocessing step, the moving points that are possibly worth tracking are detected. This is done in two steps.

The first step is foreground segmentation using a Gaussian mixture-based background/foreground segmentation algorithm (Stauffer and Grimson, 1999). The resulting foreground map is eroded and dilated in order to get a rough map of foreground objects and their close neighborhood. A grid is placed on the foreground image. In our implementation, the grid points have a fixed distance, but placing more points in smaller blobs could possibly increase the algorithm's performance on small objects. In some cases the foreground segmentation step can be omitted. The algorithm performs reasonably well with a static, fixed grid of points.

After the grid is found, the optical flow is calculated between the current and the following frame. This gives a motion vector field. The optical flow is computed by the Lucas-Kanade algorithm (Bouguet, 2000). In some cases the flow vector is miscomputed with a large error, which makes the trajectory search difficult. To filter out most of these errors, the optical flow is calculated twice: once in forward and once in backward direction. In backward direction we reverse the order of frames and use destination points calculated by the forward step as starting points. If the resulting position vector differs from the initial one by a certain measure, the flow vector would be considered invalid.

Figure 2. shows the vector field calculated in this step. Only vectors with length greater than a threshold (here: min. 1) are shown. The beginning of the vectors are marked with points.

After the flow is calculated, it is necessary to determine whether a particular point should be tracked. New points are added to the list of tracked points in two cases: if there are no tracked points near them, or if the direction of the flow vector calculated on the tracked point and the one calculated on the grid differs. This ensures that there are no unnecessary points added to the list of tracked points, but also that no important points are missed. Only points with a certain flow vector length are tracked. The limits of the vector length are determined based on the typical speed of thrown objects in the scene. 


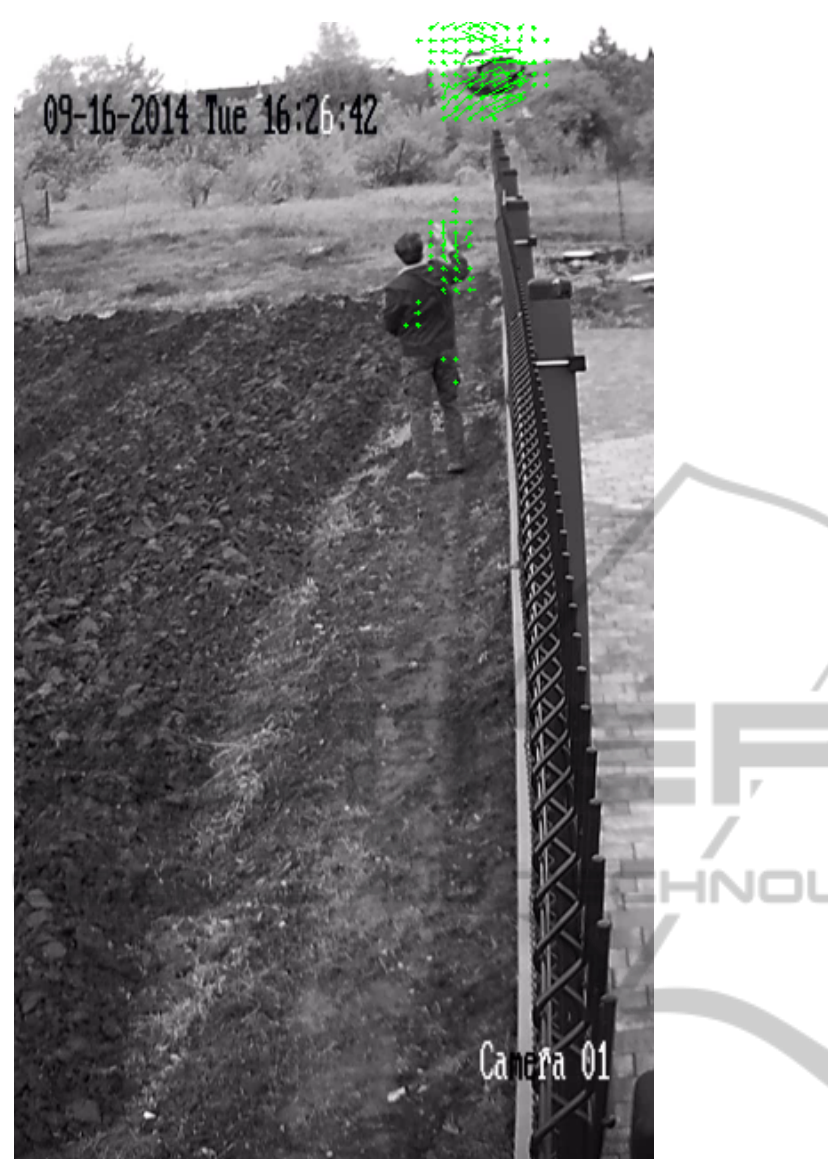

Figure 2: Detected optical flow on the foreground of the image. Only flow vectors with length greater than 1 are shown.

\subsection{Tracking Points}

The moving points detected by the previous step are added to the list of tracked points. The position history for every point is kept. At every step, the motion of the tracked points is calculated with the same dual direction optical flow algorithm used in the previous step.

The number of tracked points should be kept low in order to save resources. Practical constraints are used to discard inappropriate paths, i.e. long, straight lines are discarded. The paths which are nearly parallel with the fence are also discarded. The $x$ coordinate of the path should not change direction. The reason for this is that the trajectories of the drops should go either from left to right or from right to left while in flight. There are some kinds of motions that have near parabolic trajectories. The best example is a waving branch. These distractions are also filtered out by this method.

As a result of the object's rotation and the vari-

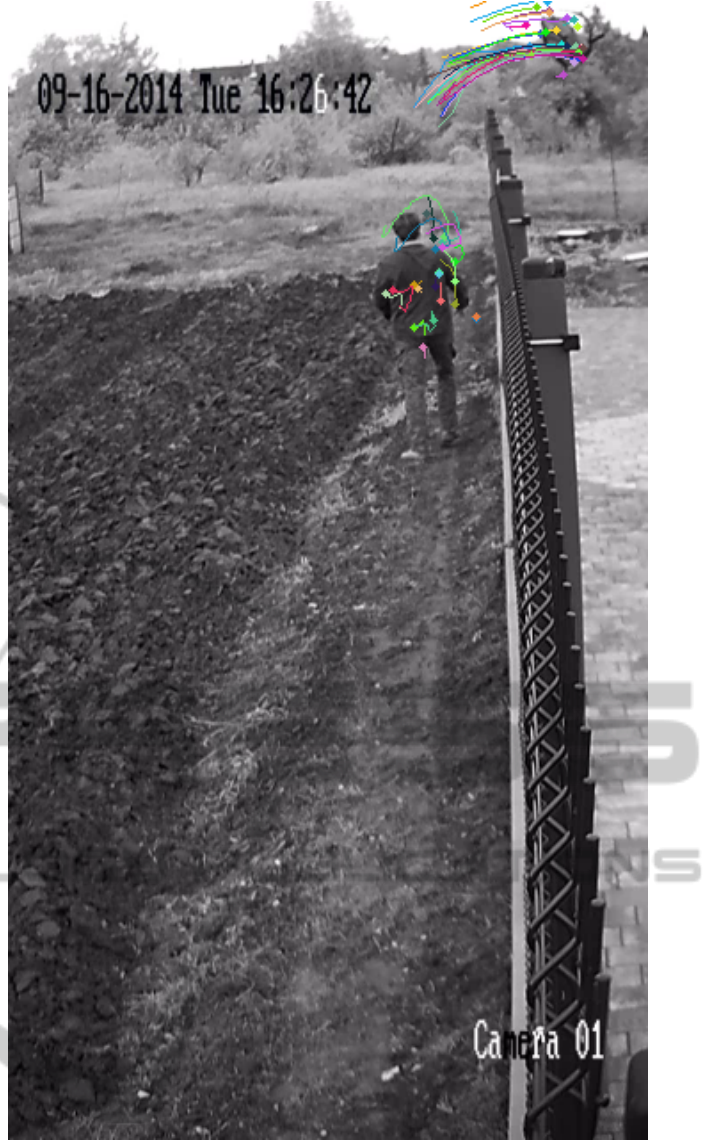

Figure 3: Trajectories of tracked points.

ability of its shape, the tracked points can be lost relatively often. When the track of a point is lost, the moving point detector detects new motion vectors in the next frame, and a new tracked point is created. This results in multiple, shorter trajectories. The trajectory matching algorithm deals with this problem.

Figure 3 shows an example of the motion history for the tracked points.

\subsection{Trajectory Matching}

Trajectory matching consists of two separate steps. The first step finds a large number of relatively small parabola fractions. This step has a large number of false positive detections. The second step is to merge these parabolas into a final parabolic path belonging to actual thrown objects.

\subsubsection{Finding Parabolic Trajectories}

The first step is trying to fit parabolas on the trajectories found by the previous step. LS fitting is used for this: a matrix is built from the coordinates of the 


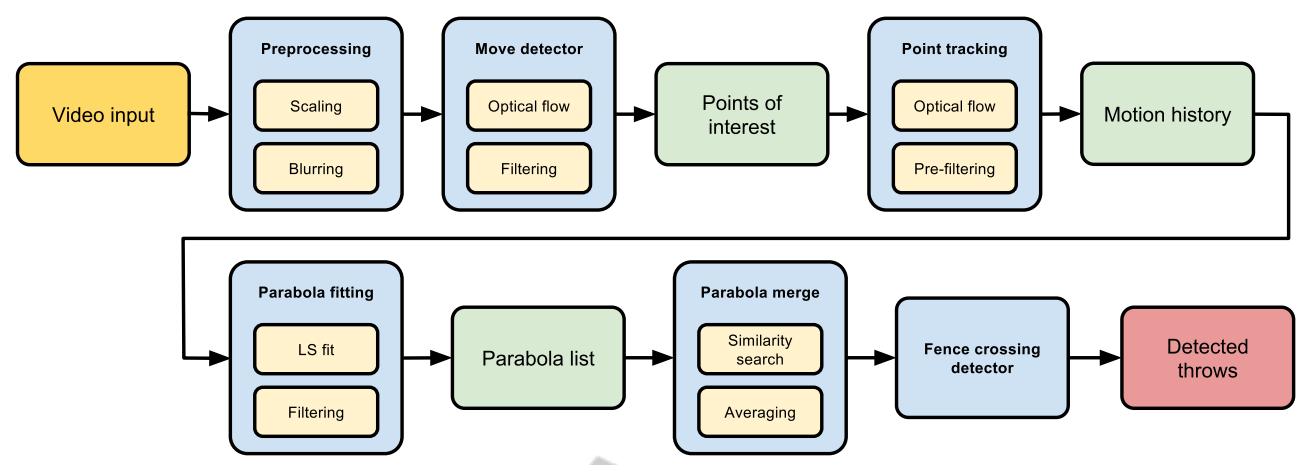

Figure 4: The structure of the system.

points on the path, and the Moore-Penrose pseudoinverse (Barata and Hussein, 2012) is taken. From this, the parameters of the parabola are easy to compute. Let us assume we have $\mathrm{N}$ points along the path. Name the coordinate pairs belonging to point $i x_{i}, y_{i}$, where $i=1 . . N$. The equation of the parabola is given by:

$$
y_{i}=a x_{i}^{2}+b x_{i}+c
$$

The unknown parameters are $a, b$, and $c$. This equation holds for every point along the path. If we want to use linear regression, we have to get rid of the $x_{i}^{2}$ part. Because the value of every $x_{i}$ is known, we can easily rename them to $z_{i}$ :

$$
z_{i}=x_{i}^{2}
$$

Define matrix $X$ as follows:

$$
X=\left[\begin{array}{ccc}
z_{1} & x_{1} & 1 \\
z_{2} & x_{2} & 1 \\
\vdots & \vdots & \vdots \\
z_{i} & x_{i} & 1 \\
\vdots & \vdots & \vdots \\
z_{N} & x_{N} & 1
\end{array}\right]
$$

vector $Y^{\top}$ as follows:

$$
Y^{\top}=\left[\begin{array}{llllll}
y_{1} & y_{2} & \cdots & y_{i} & \cdots & y_{N}
\end{array}\right]
$$

and vector $P^{\top}$ as:

$$
P^{\top}=\left[\begin{array}{lll}
a & b & c
\end{array}\right]
$$

Now we want $X \cdot P$ to be close to $Y$. We are searching for parameters $P$ that give the minimum Euclidean norm among all solutions:

$$
\min _{P}\|X \cdot P-Y\|_{2}
$$

The solution is the least-square approximation:

$$
P=X^{+} \cdot Y
$$

where $X^{+}$denotes the Moore-Penrose pseudoinverse.
The finite length parabolas have two more properties: their beginning and ending $x$ coordinate. These are just the $x$ coordinates of the leftmost and rightmost points in the position history.

After fitting a parabola to the position history, the square error of the fit is calculated at the points in the history. They are summed and normalized by the number of points, giving an error measure that is independent from the number of points. If the error is within certain limits, the trajectory is assumed to be a parabola.

There are many short trajectories found because of background clutter or other objects in the scene. To avoid an unnecessary matching step, the approximate arch length is calculated prior to the fitting process. If the length is within certain limits the fit is calculated.

In some cases the paths contain other than parabolic parts. Most of these situations arise from the fact that someone throws the object with their hand. If the camera has a clear sight of the object during the fling of arm, the points can be tracked before the object leaves the hand of the thrower. In these situations the initial part of the path is closer to an arc of a circle than to a parabola. Nevertheless, the top part of the trajectory is still a parabola after the object leaves the hand providing the initial force. In these situations a simple parabola matching fails because of a large fitting error. Thus, these situations require a special treatment: only the part that is most likely parabolic is cut out from the position history and is used for a second try of fitting a parabola. Because of free flight, the highest point of the path is most likely a part of the parabola. The maximum of the path is considered as a clear maximum if there are points on its both sides in $x$ direction. If the maximum of the path is clear, points in a window of a specific length centered around the maximum are used. If there is no clear maximum found, the window is placed at the end of the path that is closer to the top of the image. The length of the window can be approximately determined by the frame rate, the average distance, and 
the average speed of a thrown object.

The parabolic trajectories that belong to a thrown object must be oriented in the right direction: they must have a maximum and not a minimum. Therefore, the parameter $a$ should be positive (if the origin is in the bottom left corner). There is also a certain upper limit for this parameter, because the parabola cannot be very narrow. This helps to filter out some false detections.

The parabolas which passed all the tests are saved in a queue. The parameters $a, b, c, x_{\text {start }}$ and $x_{\text {stop }}$ are saved, along with the frame index of the detection which serves as a timestamp. $x_{\text {start }}$ and $x_{\text {stop }}$ defines the $x$ coordinates of the ending points of the parabola.

Figure 5 shows an example of detected parabolas after throwing a backpack through the fence.

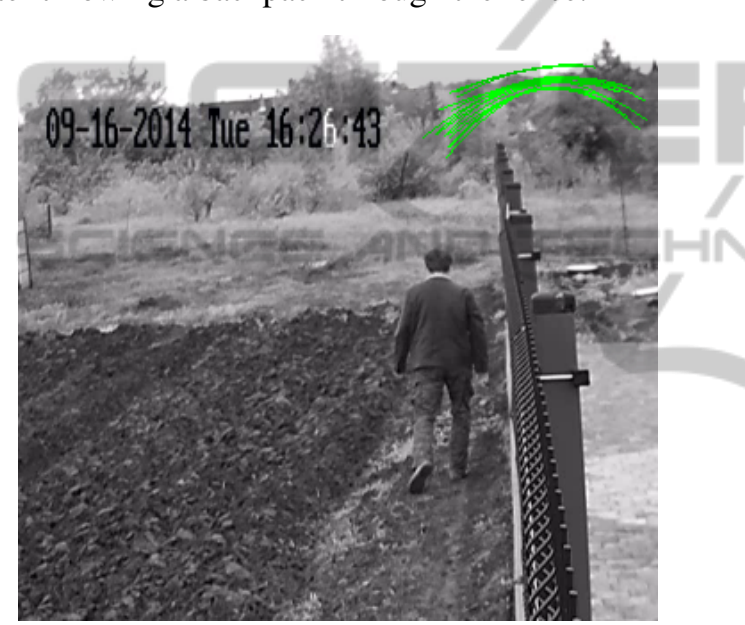

Figure 5: Parabolas found based on the motion history of tracked points.

\subsubsection{Merging Trajectories}

The final step of the algorithm is run once a parabola of a certain age is present in the list of detected parabolas. Age is determined by the associated frame index of the parabola. This introduces a small delay before the algorithm produces its final output, but it also allows for all the parabolas related to a single drop to be merged together. The delay is about $1 \mathrm{sec}-$ onds.

The main function of this step is to find similarities in the list of detected parabolas. In order to do this, a measure for parabola similarity must be defined. The parabolas that look similar and are near each other should be merged. For this, three major conditions must be met: First, the maximums of the parabolas (which are points $\left(x_{\max , i}, y_{\max , i}\right)$ ) should be near each other. The limit for the distance is determined in an empirical way. Second, the $a$ parameters should be close. Third, their base must overlap. The base of a parabola is defined as the range of $x$ values under the arc of the parabola. This criterion is easy to meet when an object is thrown: there are many parallel parabolas, so it is very unlikely that they are all disconnected in such way that no bases overlap. Yet, it helps a lot to prevent false alarms when the line of the fence is taken into account. The reason for this is that the starting and ending points are more certain.

The algorithm does the merging process in two passes, and uses heuristics for faster and easier implementation. After a parabola old enough is found, the search begins for parabolas similar to it. The average of their maximum and their $a$ parameter is calculated. The second pass uses these values to find a final set of parabolas. This eliminates the error that could arise when a the oldest parabola is one with a misdetected placement.

When similar parabolas are found, they are averaged, and the final trajectory is found. The set of similar parabolas are removed from the list.

After the final trajectory is found, an additional filtering step is applied. The starting and ending point of the parabola is connected with a straight line, and the absolute value of the angle of the line is checked. If the angle is too big, the parabola is highly asymmetric and is discarded.

\subsubsection{Considering the Line of the Fence}

If we know the line of the fence, the performance of the algorithm can be improved. In our case, we tuned the parameters of the algorithm so that it gives more false positive matches than false negatives. There is normally no motion that crosses the line of the fence. So if the detected parabola crosses the line of the fence, we can be certain that an object is thrown. Note that the line of the fence itself is not enough to make the decision: the parabola detection is important for filtering out a false event such as birds flying above the fence, or a branch of a tree wiggling in the breeze.

In steady camera surveillance systems the line of the fence is well known. The algorithm checks for line crossing by checking if the starting and ending points of the parabolas are on the opposite side of the line assigned to the fence. If they are, the trajectory is classified as a trajectory of a thrown object, and an event is generated.

Figure 6 shows the trajectory of the backpack. Bold red parabola is the final match.

\section{EXPERIMENTS}

We were able to successfully detect thrown objects in 


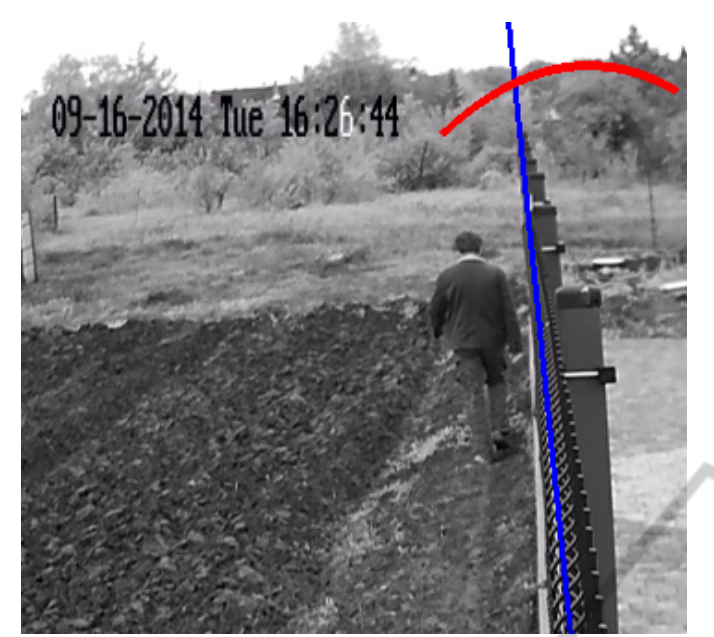

Figure 6: The detected trajectory of the thrown object.

outdoor scenes. We have not found any publicly available test sets to directly compare our solution with those of others. The existing systems are commercial, and there are no public test sets available. We made our own test set, which is available at http://almafa.org/xdever/FenceTestSets.zip. For reliable detection the object should have a minimum diameter of 30 pixels, and the stream should have a minimal frame rate of $25 \mathrm{fps}$. Note that in the test sets there are objects with smaller diameters than that.

The precision is close to $100 \%$ because considering the line of the fence and using the parabolic trajectory detection algorithm make false positives very unlikely.

Recall rates vary from scene to scene. We used 4 different test sets. They are shown in Table 1. The object used for deformable tests was a shirt. A backpack was used as rigid object.

Table 1: Test results (precision close to $100 \%$ ).

\begin{tabular}{|c|c|c|}
\hline Scene & No. of samples & Recall \\
\hline B\&W, Rigid & 9 & $90 \%$ \\
\hline Outdoor 1, Rigid & 56 & $83 \%$ \\
\hline Outdoor 1, Deformable & 38 & $73 \%$ \\
\hline Outdoor 2, Rigid & 101 & $48 \%$ \\
\hline
\end{tabular}

Throwing deformable objects also resulted in high accuracy. In contrast, scene Outdoor 2 performed poorly. The reason for this was that, on many samples, the thrown object was very small. Also, there was an artefact in the background the pattern of which was very similar to the object.

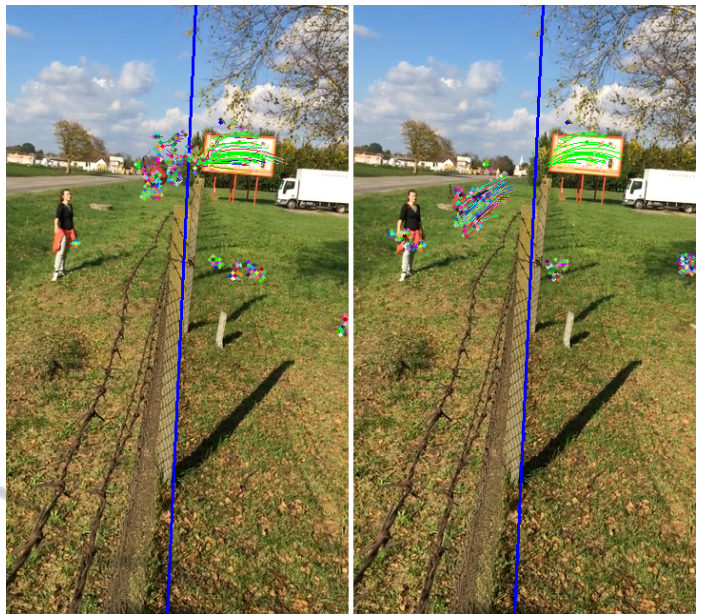

Figure 7: An example of failure of tracking the object's path.

\section{CONCLUSIONS}

The proposed algorithm performs well in outdoor scenarios, better than other methods in indoor environments (Ribnick et al., 2007). There are, however some background patterns that can interfere with the Lucas-Kanade based motion tracker. This could possibly be improved by Kalman filtering, which needs further investigation. We were also able to achieve excellent results with deformable objects. The fusion of different methods, including tracking-based and optical flow based solutions, should be investigated in order to provide better coverage of all cases. Pedestrian detection (Havasi et al., 2007) and thrown activity may be merged in a common framework.

\section{ACKNOWLEDGEMENTS}

This work has been supported by the EU FP7 Programme (FP7-SEC-2011-1) No. 285320 (PROACTIVE project). The research was also partially supported by the Hungarian Scientific Research Fund (No. OTKA 106374).

\section{REFERENCES}

Barata, J. C. A. and Hussein, M. S. (2012). The moorepenrose pseudoinverse: A tutorial review of the theory. Brazilian Journal of Physics, 42(1-2):146-165.

Bouguet, J.-Y. (2000). Pyramidal implementation of the lucas kanade feature tracker. Intel Corporation, Microprocessor Research Labs. 
Denman, S., Fookes, C., and Sridharan, S. (2009). Improved simultaneous computation of motion detection and optical flow for object tracking. In Digital Image Computing: Techniques and Applications, 2009. DICTA '09., pages 175-182.

Havasi, L., Szlavik, Z., and Sziranyi, T. (2007). Detection of gait characteristics for scene registration in video surveillance system. IEEE Trans Image Processing, 16(2):503-510.

Ribnick, E., Atev, S., Papanikolopoulos, N., Masoud, O., and Voyles, R. (2007). Detection of thrown objects in indoor and outdoor scenes. In Intelligent Robots and Systems, 2007. IROS 2007. IEEE/RSJ International Conference on, pages 979-984.

Stauffer, C. and Grimson, W. (1999). Adaptive background mixture models for real-time tracking. In Computer Vision and Pattern Recognition, 1999. IEEE Computer Society Conference on., volume 2, pages -252 Vol. 2.

Venetianer, P., Lipton, A., Chosak, A., Frazier, M., Haering, N., Myers, G., Yin, W., and Zhang, Z. (2005). Video surveillance system. US Patent App. 11/057,154. 